\title{
UNIVERSAL BEHAVIOUR OF MAGNETOCONDUCTANCE DUE TO WEAK LOCALIZATION IN TWO-DIMENSIONAL SYSTEMS - EXAMPLE OF GaInAs QUANTUM WELLS
}

\author{
A. ZduniaK* ${ }^{*}$, M.I. Dyakonov ${ }^{\dagger}$, E. Litwin-STaszewska ${ }^{\ddagger}$ AND W. KNaP \\ Groupe d'Etudes des Semiconducteurs, Université de Montpellier II \\ 34095 Montpellier, France
}

Weak localization corrections to conductivity of a two-dimensional electron gas are studied by measurements of the magnetic field dependence of the conductivity in GaInAs quantum wells. We observe that, when presented as a function of the normalized magnetic field $\left(x=B / B_{\mathrm{tr}}\right.$, where $B$ is the magnetic field, $B_{\mathrm{tr}}=\hbar / 4 e \tau D, D$ is the diffusion constant and $\tau$ is momentum relaxation time), different samples show very similar high field behaviour. A theoretical description is developed that allows one to describe in a consistent way high and low field behaviour. The theory predicts universal $\left(B^{-1 / 2}\right)$ behaviour of the conductivity correction for all 2D systems in the high field limit $(x>1)$. Low field behaviour depends strongly on spin and phase relaxation mechanisms. Comparison of the theory with experiment confirms the universal behaviour in the high field limit and allows one to estimate the spin and phase relaxation times for different GaInAs quantum wells.

PACS numbers: 73.20.Dx, 73.61.Ey

\section{Theoretical background}

Weak-field magnetoconductance is due to quantum corrections to the conductivity, $\Delta \sigma$, arising from interference of electron waves scattered along closed paths in opposite directions. This interference is destroyed by the magnetic field because of the phase shift between the corresponding amplitudes which is equal to $2 \pi \phi / \phi_{0}$, where $\phi=B S$ is the magnetic flux through the area $S$ of a closed path, $\phi_{0}=\pi \hbar / e$ is the elementary flux quantum. This idea lies in the basis of the theory of the weak localization magnetoconductance $[1,2]$.

\footnotetext{
* On leave from Physics Department of Warsaw University, 02-658 Warszawa, Poland.

tOn leave from A.F. Ioffe Physico-Technical Institute, 194021 St. Petersburg, Russia.

tOn lea ve from High Pressure Research Center, Polish Academy of Sciences, 01-142 Warszawa, Poland.
} 
To describe the magnetic field dependence of the quantum correction to conductivity three characteristic field values are usually introduced: $B_{\phi}, B_{\mathrm{so}}$ and $B_{\mathrm{tr}}$, where

$$
B_{\phi}=\frac{\hbar}{4 e D \tau_{\phi}}, \quad B_{\mathrm{so}}=\frac{\hbar}{4 e D \tau_{\mathrm{s}}}, \quad B_{\mathrm{tr}}=\frac{\hbar}{4 e D \tau} .
$$

Here $D=l^{2} / 2 \tau$ is the diffusion coefficient, $l=v_{\mathbf{F}} \tau$ is the mean free path, $v_{\mathbf{F}}$ is the Fermi velocity, $\tau, \tau_{\mathrm{s}}$, and $\tau_{\phi}$ are the elastic scattering, the spin relaxation, and the phase-breaking times, respectively.

It is convenient to use the expression for the quantum correction to the conductivity derived in Ref. [1], which may be written as

$$
\begin{aligned}
& \Delta \sigma(B)=-\frac{e^{2}}{2 \pi^{2} \hbar} F(x), \quad F(x)=x \sum_{n=0}^{\infty} \frac{P_{n}^{3}}{1-P_{n}}, \\
& P_{n}=s \int_{0}^{\infty} \mathrm{d} t \exp \left(-s t-t^{2} / 2\right) L_{n}\left(t^{2}\right),
\end{aligned}
$$

where $s=(2 / x)^{1 / 2}, L_{n}$ is the $n$-th Laguerre polynomial.

It is easy to take account for the phase breaking and spin-relaxation processes and thus to obtain a complete formula describing the quantum correction to conductivity not only in the high field limit, $\left(B \gg B_{\phi}, B_{\mathrm{so}}\right)$, as Eqs. (2), (3), but also in the low field limit $\left(B<B_{\phi}, B_{\mathrm{so}}\right)$. If, for a moment, we consider phase-breaking processes only, an additional factor $\exp \left(-\left|r-r^{\prime}\right| / l_{\phi}\right)$ should be introduced into the relation which is used to calculate $W(r)$ - the probability to find an electron in the area $\mathrm{d}^{2} r$ around the point $r$ (see Ref. [1]), where $l_{\phi}=v_{\mathrm{F}} \tau_{\phi}=l \tau_{\phi} / \tau$. This leads to the following modification of the expression for $P_{n}$ in Eq. (3):

$$
P_{n}=s \int_{0}^{\infty} \mathrm{d} t \exp \left[-s\left(1+\beta_{\phi}\right) t-t^{2} / 2\right] L_{n}\left(t^{2}\right),
$$

where $\beta_{\phi}=\tau / \tau_{\phi}=B_{\phi} / B_{\mathrm{tr}}$. We denote by $F\left(x, \beta_{\phi}\right)$ the function defined as $F(x)$ in Eq. (2), but with $P_{n}$ modified according to Eq. (4). Thus, if spin relaxation is ignored

$$
\Delta \sigma(B)=-\frac{e^{2}}{2 \pi^{2} \hbar} F(x, \beta) .
$$

We now introduce spin relaxation. This may be done by a simple generalization of the Ilikami-Larkin-Nagaoka (HLN) results [3]. As it is well known (see Refs. $[2,3]$ ) the quantum correction may be split into a positive singlet part, which is not influenced by spin relaxation (in the absence of magnetic impurities), and the negative triplet part which depends on the spin relaxation rate. In the $2 \mathrm{D}$ case, when the fluctuating magnetic field responsible for spin relaxation lies in the 2D plane, the two in-plane spin components relax with the same time constant, $\tau_{\mathrm{s}}$, while the normal to the plane spin component is destroyed two time faster. These considerations lead to the following formula which accounts for the phase-breaking and spin relaxation processes and is applicable for arbitrary magnetic fields (limited only by the condition $\omega_{\mathrm{c}} \tau \ll 1$, where $\omega_{\mathrm{c}}$ is the cyclotron frequency):

$$
\Delta \sigma(B)=-\frac{e^{2}}{2 \pi^{2} \hbar}\left[F\left(x, \beta_{s 1}\right)+\frac{1}{2} F\left(x, \beta_{s 2}\right)-\frac{1}{2} F\left(x, \beta_{\phi}\right)\right],
$$


where

$$
\beta_{s 1}=\frac{B_{\phi}+B_{\mathrm{so}}}{B_{\mathrm{tr}}}, \quad \beta_{s 2}=\frac{B_{\phi}+2 B_{\mathrm{so}}}{B_{\mathrm{tr}}}, \quad \beta_{\phi}=\frac{B_{\phi}}{B_{\mathrm{tr}}} .
$$

The quantities $B_{\phi}, B_{\mathrm{so}}$, and $B_{\mathrm{tr}}$ are defined by Eq. (1), where $\tau_{\mathrm{s}}$ should be understood as the relaxation time for the in-plane spin components . For $^{2}=B / B_{\mathrm{tr}} \ll 1$ Eq. (6) reduces to the HLN formula [3]

$$
\begin{aligned}
\Delta \sigma(B)= & -\frac{e^{2}}{2 \pi^{2} \hbar}\left[\Psi\left(\frac{1}{2}+\frac{1}{x}\right)-\Psi\left(\frac{1}{2}+\frac{\beta_{s 1}}{x}\right)+\frac{1}{2} \Psi\left(\frac{1}{2}+\frac{\beta_{\phi}}{x}\right)\right. \\
& \left.-\frac{1}{2} \Psi\left(\frac{1}{2}+\frac{\beta_{s 2}}{x}\right)\right],
\end{aligned}
$$

where $\Psi$ is the di-gamma function. For large $x$ the universal curve given by Eqs. (2), (3) is recovered.

Some remarks are in order, concerning the notion of universality for the high field behaviour of the quantum correction $\Delta \sigma$. It is universal in the sense that for $B \gg B_{\phi}, B_{\mathrm{so}}$ and for a given elastic scaltering mechanism $\Delta \sigma$ depends on magnetic field only through the parameter $x=B / B_{\mathrm{tr}}$. For the case of isotropic scattering (short-range impurity potential) this dependence was calculated in Ref. [1], and this is the case for which the formulas given above were derived.

\section{Experimental results}

Four AlGaAs/InGaAs/GaAs pseudomorphic quantum wells were used for the experiment. They were grown by the molecular beam epitaxy technique. The two-dimensional electron gas was formed in the $13 \mathrm{~nm}$ thick InGaAs layer. All samples were $\delta$-doped with Si (doping density $N=2.5 \times 10^{12} \mathrm{~cm}^{-2}$ ). They had AlGaAs spacer of thickness from $2 \mathrm{~nm}$ to $6 \mathrm{~nm}$. After slow cooling (to $4.2 \mathrm{~K}$ ) in the dark the sample with the biggest spacer had the lowest carriers concentration in InGaAs layer $\approx 1.05 \times 10^{12} \mathrm{~cm}^{-2}$, the samples with $4 \mathrm{~nm}$ spacer $\approx 1.35 \times 10^{12} \mathrm{~cm}^{-2}$ and the sample with $6 \mathrm{~nm}$ spacer $\approx 1.6 \times 10^{12} \mathrm{~cm}^{-2}$. Illumination by infra-red emitting diode allowed us to increase persistently the carriers concentration in the range of about $20 \%$ for each sample. The behaviour of the samples under infra-red illumination was caused by metastable properties of DX-Si centres present in $\mathrm{AlGaAs}$ layer.

To generate stable weak magnetic field sweep necessary for weak localization measurements we used a system of two superconducting coils $(80 \mathrm{kG} / 80 \mathrm{kG})$ placed in the same cryostat. Slowly tuning spread field of the upper coil was compensating constant field in the bottom one. Typically the constant magnetic field in the bottom (sample) coil was of the order of 50 gauss and the upper coil field was tuning in the range from 0 to $80 \mathrm{kG}$.

For the case of degenerated 2D electron gas the diffusion constant can be expressed as $D=\frac{1}{2}\left(\hbar k_{\mathrm{F}} / m^{*}\right)^{2} \tau$, where $k_{\mathrm{F}}$ is the Fermi vector, $m^{*}$ is the effective

${ }^{5}$ We take this occasion to note that the quantities $\tau_{\mathrm{so}}^{(x)}, \tau_{\mathrm{so}}^{(y)}, \tau_{\mathrm{so}}^{(z)}$ introduced and defined in the original HLN paper are not the relaxation times of the corresponding spin components. This notation has caused some confusion in the literature. The time $\tau_{s}$ which we introduced here, is the relaxation time for the two in-plane spin components, while the relaxation time for the normal to the plane is $\tau_{s} / 2$. 
mass of free carriers. In this case the Fermi vector $k_{\mathrm{F}}=2 \pi N_{\mathrm{s}}\left(N_{\mathrm{s}}\right.$ is the carriers concentration). Using Eq. (1) and above formulas leads to

$$
B_{\mathrm{tr}}=\frac{e}{2 h N_{\mathrm{s}} \mu^{2}} \text {. }
$$

After each illumination Shubnikov-de Haas measurements were performed to establish $N_{\mathrm{s}}$ value. To calculate $B_{\mathrm{tr}}$ parameter zero magnetic field mobility was used. The $B_{\mathrm{tr}}$ parameter derived from Eq. (9) changes from 20 gauss to 50 gauss for our samples. The experimental system is described in more details in Ref. [4].

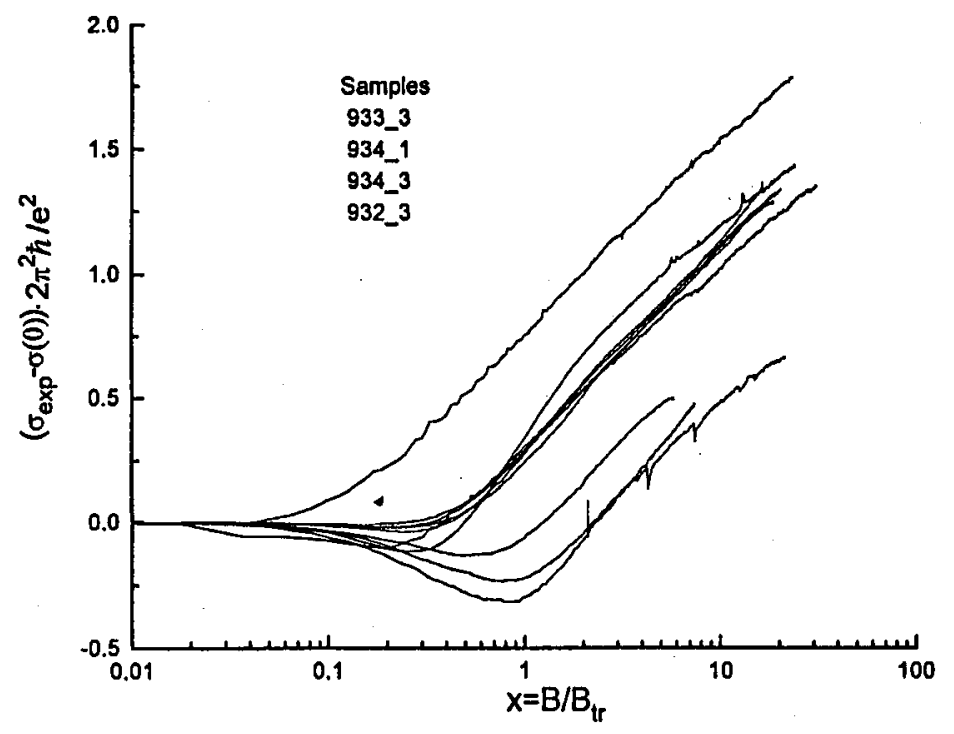

Fig. 1. Magnetoconductivity of GaInAs quantum wells presented as a function of normalized magnetic field. Universal behaviour is seen as parallelity of the curves for $x>1$.

Figure 1 shows measurements of magnetoconductivity presented as a function of normalized magnetic field $\left(\boldsymbol{x}=B / B_{\mathrm{tr}}\right)$ for various values of $B_{\mathrm{tr}}$ (i.e, for various samples and various rates of illumination). The universal behaviour of magnetoconductivity manifests here by the fact that different curves are almost parallel for $x=B / B_{\mathrm{tr}}$ greater than one. For smaller values of $x$ magnetoconductivity depends strongly on spin and phase breaking mechanisms. This explains why the curves for $x<1$ are not parallel.

In Fig. 2 we present the fits of formula (6) for two of our experimental results. One can see that both low field and high field behaviour is well reproduced. The fitted values of ratio $\tau_{\phi} / \tau$ and $\tau_{s} / \tau$ are marked on the picture. We found that for our samples $\tau_{\phi} / \tau$ changes in the range from 35 to 50 , and $\tau_{\mathrm{s}} / \tau$ in the range from 15 to 35 . Because $\tau$ is determined from the zero field mobility and the known effective mass [5] we calculated that the values of $\tau_{\phi}$ change in the range $35-50 \mathrm{ps}$ and $\tau_{\mathrm{s}}$ changes in the range $15-35 \mathrm{ps}$.

It is important to note that for $x>1$ experimental data follow similar functional dependence approaching the theoretically predicted universal behaviour. 


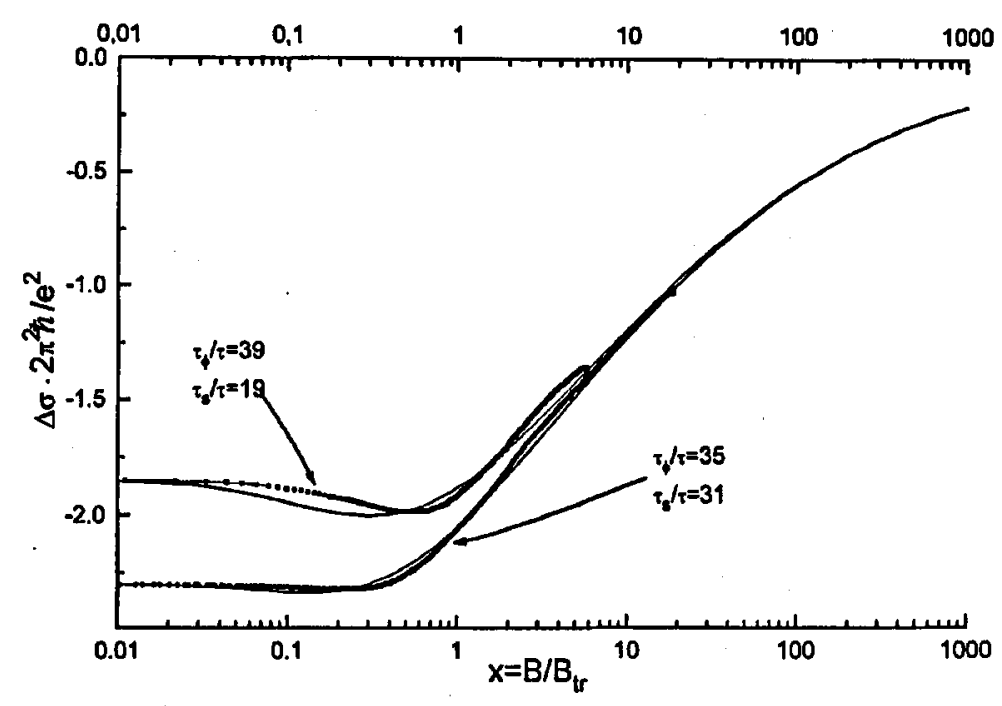

Fig. 2. Example of fit of formula (6) for whole range of magnetic fields. Estimated values of spin and phase relaxation times are given.

\section{Conclusion}

Weak localization corrections to conductivity of a 2D electron gas in GaInAs quantum wells were studied. Theoretical model allowing a consistent description of low $(x<1)$ and high $(x>1)$ magnetic field was presented. It allowed one to estimate spin and phase relaxation times for examined structures. For $x>1$ results from different samples approach the theoretically predicted universal behaviour.

\section{Acknowledgments}

We wish to thank K. Zekentes from Forth Institiute (Heraklion, Greece) for growing samples. Discussions with M. Grynberg, J.L. Robert, T. Dietl and V. Mosser are gratefully acknowledged.

\section{References}

[1] M.I. Dyakonov, Solid State Commun. 92, 711 (1994).

[2] B.L. Altshurer, A.G. Aronov, D.E. Khmelnitskii, A.I. Larkin, in: Quantum Theory of Solids, Ed. I.M. Lifshits, MIR Publishers, Moscow 1982.

[3] S. Hikami, A.I. Larkin, Y. Nagaoka, Prog. Theor. Phys. 63, 781 (1980).

[4] W. Knap, D. Dur, A. Raymond, C. Meny, J. Leotin, S. Huant, B. Etienne, Rev. Sci. Instrum. 63, 3293 (1992).

[5] E. Litwin-Staszewska, F. Kobbi, M. Kamal-Saadi, D. Dur, C. Skierbiszewski, H. Sibari, K. Zekentes, V. Mosser, A. Raymond, W. Knap, J.L. Robert, Solid State Electron. 37, 665 (1994). 